\title{
Label-free SERS detection of Raman-Inactive protein biomarkers by Raman reporter indicator: Toward ultrasensitivity and universality
}

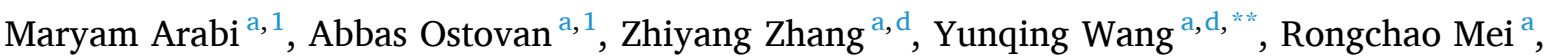 \\ Longwen $\mathrm{Fu}^{\mathrm{a}}{ }^{\mathrm{a}}$, Xiaoyan Wang ${ }^{\mathrm{a}, \mathrm{b}}$, Jiping Ma ${ }^{\mathrm{c}}$, Lingxin Chen ${ }^{\mathrm{a}, \mathrm{d}, *}$ 1xchen@yic.ac.cn \\ ${ }^{a}$ CAS Key Laboratory of Coastal Environmental Processes and Ecological Remediation, Shandong Key Laboratory of Coastal Environmental Processes, Yantai Institute of \\ Coastal Zone Research, Chinese Academy of Sciences, Yantai, 264003, China \\ ${ }^{\mathrm{b}}$ School of Pharmacy, Binzhou Medical University, Yantai, 264003, China \\ c School of Environmental \& Municipal Engineering, Qingdao University of Technology, Qingdao, 266033, China \\ d Center for Ocean Mega-Science, Chinese Academy of Sciences, Qingdao, 266071, China
}

\section{A R T I C L E I N F O}

\section{Keywords:}

Surface-enhanced Raman scattering

Raman reporter indicator mechanism Capillary sensor

Mussel-inspired molecular imprinting

Bioassay

\begin{abstract}
A B S T R A C T
It is still challenging to sensitively detect protein biomarkers via surface-enhanced Raman scattering (SERS) technique owing to their low Raman activity. SERS tag-based immunoassay is usually applied; however, it is laborious and needs specific antibodies. Herein, an ultrasensitive and universal "Raman indicator" sensing strategy is proposed for protein biomarkers, with the aid of a glass capillary-based molecularly imprinted SERS sensor. The sensor consists of an inner SERS substrate layer for signal enhancement and an outer mussel-inspired polydopamine imprinted layer as a recognition element. Imprinted cavities have two missions: first, selectively capturing the target protein, and second, the only passageway of Raman indicator to access SERS substrate. Specific protein recognition means filling imprinted cavities and blocking Raman indicator flow. Thus, the quantity of captured protein can be reflected by the signal decrease of ultra-Raman active indicator molecule. The capillary sensor exhibited specific and reproducible detection at the level down to $4.1 \times 10^{-3} \mu \mathrm{g} \mathrm{L}^{-1}$, for trypsin enzyme in as-received biological samples without sample preparation. The generality of the mechanism is confirmed by using three different protein models. This platform provides a facile, fast and general route for sensitive SERS detection of Raman inactive biomacromolecules, which offers great promising utility for in situ and fast point-of-care practical bioassay.
\end{abstract}

\section{Introduction}

Nowadays, analytical detection/monitoring of biomarkers receives increasing concerns due to high demand in numerous dynamic areas of life sciences, biotechnology, and health care (Kumar et al., 2019; Shetti et al., 2019b). However, bioanalytes have low abundance in biological samples often beyond the detection limit of high-tech instruments and accompanied by high concentration coexisting interfering species. So far, intelligent fashions have developed to tackle the unmet challenges of bioanalysis. Recently, designing sensor platforms for fast, low-cost, and on-site detection of different bioanalytes has attracted significant interest (Shetti et al. 2019a, 2020). The systems rely on immunoassay have been in the spotlight of attention owing to the dominant merit of high specificity (Li et al., 2019a; Moura et al., 2020). Unfortunately, the requirement of qualified specific antibodies is inevitable, which makes these methods expensive, toilsome, and time-consuming. Moreover, antibodies have poor reproducibility, instability, and sometimes cannot be generated for target species (Baker 2015a, b). Such imperfections have limited the broad application of antibodies, especially in areas that laboratory facilities and funding are scarce like rural regions.

Molecularly imprinted polymers (MIPs) as artificial receptors containing antibody mimic molecular recognition sites can be generated by

\footnotetext{
* Corresponding author. CAS Key Laboratory of Coastal Environmental Processes and Ecological Remediation, Yantai Institute of Coastal Zone Research, Chinese Academy of Sciences, Yantai, 264003, China.

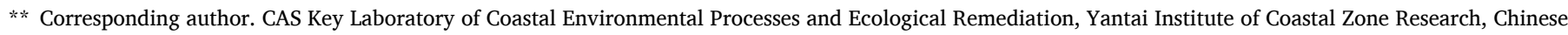
Academy of Sciences, Yantai, 264003, China

E-mail address: yqwang@yic.ac.cn (Y. Wang).

1 These authors contributed equally: Maryam Arabi, Abbas Ostovan.
} 
the templating technique in synthetic polymers' network. Created threedimensional imprinted cavities are spatiality complementary to the shape, size and functionality of the target analyte. Simple synthesis route, high stability in the harsh conditions and long storage, and costefficient properties of MIPs cause these receptors to be an eligible alternative of natural antibodies (Chen et al., 2016; Yang et al., 2018). However, the crucial challenges have been faced during the construction of MIPs-based platforms, operational and the sensing process for bioassay. For the early diagnosis of a disease, the key point is specific measuring of bioanalytes at an ultra-trace quantity, which requires a highly sensitive and selective sensing mechanism. Coupling MIPs with a sensitive detection scheme has dramatically accomplished this mission.

Among operational detection schemes, surface-enhanced Raman scattering (SERS) exhibits great potential in clinical diagnosis due to minor susceptibility to the environmental variables, miniature/portable instruments for in-situ detection and offering highly sensitive, timely, accurate, and nondestructive information (Kao et al., 2020; Lim et al., 2020). Two major deficiencies of SERS namely designing targets capturing interfaces and resisting the matrix interference remarkably overcome by immobilization MIP units on the SERS active substrate. In MIP-SERS technique, MIP assures the selectivity of a sensor and SERS renders the sensitivity (Guo et al., 2020; Li et al., 2019b). Up to now, two main sensing mechanisms have been developed for MIP-SERS sensors (Fig. S1): I) direct analyte capturing and sensing; it may not present desirable sensitivity because of the intrinsic low Raman signal of the analyte. II) Detection with the aid of SERS nanotags. Although upgraded formats of the second technique termed boronate affinity sandwich assay (Usta et al., 2016; Ye et al., 2014) and plasmonic immunosandwich assay (Muhammad et al., 2017; Xing et al., 2019) offered high sensitivity, it is laborious to be performed; also the analyte is limited to large biomolecules with multiple (at least two) binding sites, one site for MIP and the other site for the antibody of the SERS nanotag.

Herein, a novel ultrasensitive and universal "Raman indicator" sensing strategy is proposed for protein detection, with the aid of mussel-inspired surface-imprinted capillary SERS sensor. In the proposed Raman indicator sensing mechanism, recognition reaction between surface imprinted polymer and the target protein is accompanied by blocking the flux of ultrasensitive Raman reporter indicator towards the SERS active substrate, thus causing SERS signal decreasing. By relying on the advantages of Raman indicator sensing mechanism, crude biological samples can be directly analyzed without needing any dilution or treatment within $15 \mathrm{~min}$, which demonstrated the high practical impact of the proposed sensor. Besides, sensing operation can be easily performed by the untrained operator since it just involves dipping MIPSERS sensor in corresponding solutions. Through this strategy, the sensitivity greatly enhanced and offers detection limit down to $4.1 \times$ $10^{-3} \mu \mathrm{g} \mathrm{L}^{-1}$ for trypsin enzyme model. In addition, the high generality of the proposed sensing mechanism is confirmed by using bovine serum albumin (BSA), pepsin (PS), and hemoglobin (HG) as bioanalyte models. The capillary sensor as highly promising micro-platform sensing has wide applications, particularly in clinical diagnosis.

\section{Experimental section/methods}

\subsection{Materials and instruments}

The information of materials and instruments are available in Supporting Information.

\subsection{Synthesis of gold nanostars (Au NSs)}

Au NSs were synthesized by a facile and scalable method according to the previous report (Xie et al., 2007). Briefly, $100 \mathrm{mM}$ aqueous standard solution of HEPES was prepared with ultrapure water, and $\mathrm{pH}$ was adjusted to $7.5 \pm 0.5$ at room temperature by adding $1 \mathrm{M} \mathrm{NaOH}$ solution. $2 \mathrm{~mL}$ of $100 \mathrm{mM}$ HEPES (pH 7.5) solution was mixed with $3 \mathrm{~mL}$ of ultrapure water, followed by the addition of $40 \mu \mathrm{L} \mathrm{HAuCl}_{4}$ solution with the concentration of $24.25 \mathrm{mM}$. The solution was remained at room temperature without shaking, and the color of it was changed from light yellow to colorless, light gray, purple, and finally to blue, within $20 \mathrm{~min}$.

\subsection{Preparation of SERS-Active glass capillaries}

Both tips of glass capillaries were sealed by flame and then submerged them in RBS ${ }^{\mathrm{TM}} 25$ solution under sonication at $80^{\circ} \mathrm{C}$ for $20 \mathrm{~min}$. Then, the capillaries were washed with a high amount of ultrapure water repeatedly followed by immersing in $\mathrm{H}_{2} \mathrm{SO}_{4}-\mathrm{H}_{2} \mathrm{O}_{2}(7: 3, \mathrm{v} / \mathrm{v})$ solution and remained at room temperature for $4 \mathrm{~h}$. The capillaries again were rinsed with a high amount of ultrapure water and dried at $75{ }^{\circ} \mathrm{C}$ overnight. For amino functionalization, clean glass capillaries were vertically immersed in a $1 \%(\mathrm{v} / \mathrm{v})$ ethanol solution of APTES in anhydrous ethanol at $70{ }^{\circ} \mathrm{C}$ for $6 \mathrm{~h}$. After silanization, capillaries sonicated and rinsed three times with ethanol to remove unreacted silane, and baked for $2 \mathrm{~h}$ at $100{ }^{\circ} \mathrm{C}$ in an air oven. At the next stage, the silanized glass capillaries were dipped into the Au NSs colloidal suspension for $9 \mathrm{~h}$ to prepare $\mathrm{Au}$ NSs layer (this layer can be seen by naked eyes). Finally, glass substrates were all washed with ultrapure water for three times and dried for $30 \mathrm{~min}$ at $30^{\circ} \mathrm{C}$. SERS-Active glass capillaries were thus simply synthesized.

\subsection{Capillary SERS sensor construction based on mussel-inspired surface imprinting}

For the preparation of the imprinted layer, $10 \mathrm{mg}$ trypsin and $40 \mathrm{mg}$ dopamine hydrochloride were dissolved in $10 \mathrm{mM}$ Tris-HCl buffer ( $\mathrm{pH}$ 8.5). Afterward, SERS-Active glass capillaries were vertically dipped in the above solution and remained at room temperature for $5 \mathrm{~h}$ to complete the polymerization. Finally, trypsin molecules were removed from the PDA network by washing capillary sensor with a $3 \%(\mathrm{v} / \mathrm{v})$ solution of acetic acid containing $10 \%(\mathrm{w} / \mathrm{v})$ sodium dodecyl sulfate (SDS) for five times, and then thoroughly washed with ultrapure water to completely remove residual of acetic acid and SDS. BSA, PS, HG sensors were prepared with the same protocol. The mass ratios of PS/dopamine, BSA/ dopamine, and HG/dopamine were $10 / 100 \mathrm{mg} / \mathrm{mg}$ and imprinting times were 5,5 , and $10 \mathrm{~h}$, respectively. The control sensors were constructed in the same manner as their corresponding MIP sensor but without the addition of the template.

\subsection{Optimization}

Several experimental variables which have a significant influence on protein sensing system performance, such as mass ratios of trypsin/ dopamine, type of porogen, washing composition and duration time, the concentration of the Raman reporter and the sensor response time were optimized. The related explanation is presented in the Supporting Information.

\subsection{Bioassay procedure}

The protein measurement was performed by four simple steps involved trypsin recognition, CBBG staining, DTTC smearing followed by SERS detection. The tip of the capillary sensor dipped into a $50 \mu \mathrm{L}$ trypsin deionized water solution or a raw spiked urine sample (without any pretreatment operations such as filtration, dilution, $\mathrm{pH}$ adjustment, etc.) for $10 \mathrm{~min}$. Afterward, the sensing zone of the capillary plunged into the ultrapure water to avoid the non-specific adsorption. Subsequently, staining bonded protein by plunging the tip of the sensor in a $0.6 \mathrm{mM}$ CBBG solution. Finally, the tip of the capillary sensor marked with a $0.1 \mathrm{mM}$ DTTC solution and then analyzed with the Raman microscope. For assay of BSA, PS, HG proteins, operation steps, were same as for trypsin. For practical application of the capillary sensor, urine, saliva, and sweat samples collected from male/female healthy 
volunteers and used directly. Because saliva and sweat samples contain small tiny particles, they were filtered through a $0.22 \mu \mathrm{m}$ PES filter membrane. All samples were free of trypsin demonstrated the volunteers' physical health (SEE and SMITH 1991).

\section{Results and discussions}

\subsection{Raman indicator sensing mechanism}

Fig. 1A displays the preparation of the MIP-SERS sensor. Au NSs electrostatically anchored onto the amino-functionalized glass capillary and form SERS substrates. Then, the polydopamine (PDA) imprinted layer containing protein template uniformly covered Au NSs. Upon washing the templates, selective recognition cavities formed on the sensor surface. Fig. 1B demonstrates the Raman indicator sensing mechanism. The recognition cavities are not affected when the capillary MIP-SERS sensor is exposed to sample without target analyte (blank), and DTTC Raman reporter can easily penetrate through the vacant imprinted cavities, reach SERS substrate and generate strong "reference signal". By contrast, in a sample solution containing target protein, the recognition cavities are selectively occupied by the target protein. This incident blocks the only pathway of Raman reporter indicator flux for approaching the SERS substrate, resulting in a decrease of the SERS signal of the Raman reporter. Therefore, the amount of captured target protein can be quantitatively reflected by the change of "sensing signal" comparing with the "reference signal".

This label-free SERS sensing mechanism benefits from four key merits. 1) Selectivity. The imprinted PDA layer is responsible for
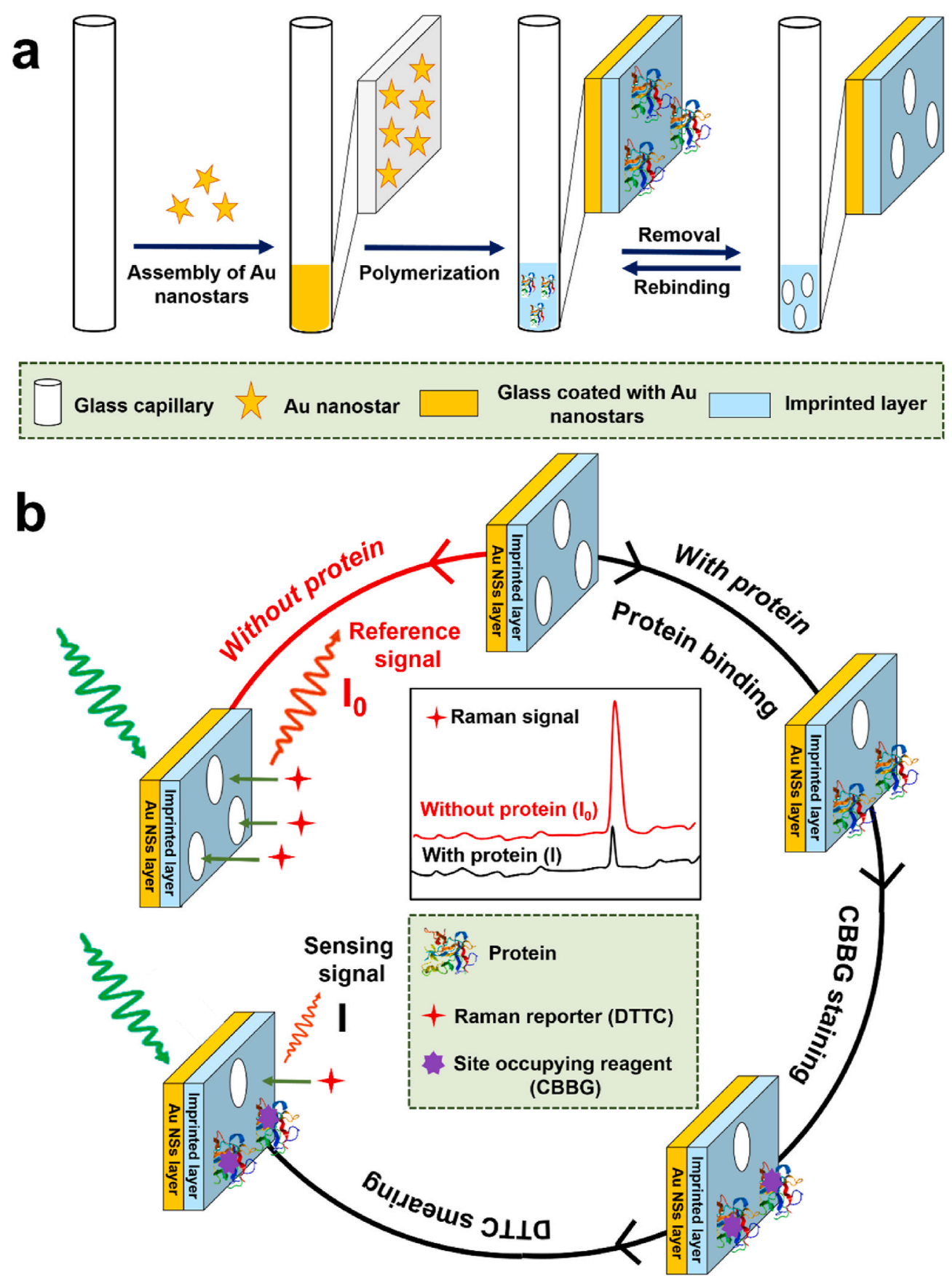

Fig. 1. a) Schematic illustration (not drawn to scale) of the capillary sensor construction by electrostatically assisted surface-assembly of Au NSs and mussel-inspired surface imprinting technique. b) Raman reporter indicator mechanism for SERS sensing of protein. 
selectivity. It is largely demonstrated that oxidation and polymerization of dopamine in an aerobic and alkaline media at the attendance of bioanalytes renders biocompatible multifunctional polymer segment with imprinted cavities (Chen et al., 2019; Lee et al., 2007b). PDA surface imprinting formed a highly cross-linked network to warrant the conservation of imprinted cavities and provide accessible binding sites with high specificity (Chen et al., 2015; Pan et al., 2018). 2) Sensitivity. Different from direct sensing of captured Raman-inactive proteins that provides weak SERS signal, this sensor indirectly senses proteins by transducing protein recognition reaction into signal decreasing of the ultrasensitive Raman reporter indicator. To attain the highest sensitivity, the combination of Au NSs substrate and 3.3'-Diethylthiatricarbocyanine iodide (DTTC) Raman reporter was selected since Au NSs possess strong SERS enhancement ability and DTTC has a large Raman scattering cross-section. This combination is helpful to gain a sensitive surface-enhanced resonant Raman scattering (SERRS) effect under a $780 \mathrm{~nm}$ laser irradiation. 3) Generality. To prevent the undesirable adsorption of DTTC by hydrophobic pockets within bonded protein molecules inside the imprinted cavities and induce background SERS signal, a Coomassie brilliant blue G-250 (CBBG) staining step was performed prior to DTTC incubation. This general protein staining reagent with a strong affinity to proteins can occupy possible binding pockets of protein targets instead of DTTC in advance (Han et al. 2010a, 2010b), thus eliminates the background SERS signal effectively. This ingenious design contributes to the analysis of proteins with varied types and sizes. 4) Fast and easy operation. No sample-pretreatment is required and the operation step can be simply performed by dipping capillary sensor in the corresponding solutions. Moreover, due to the low thickness of the MIP layer, binding of protein molecules to the specific imprinted cavities occurred swiftly. In addition to decreasing the time of analysis, fast recognition prevents transformation protein' conformation and structure (Culver and Peppas 2017; Yang et al., 2019).

\subsection{Construction and characterization of capillary sensors}

The sensor was constructed on the outer surface of the glass capillary to benefit from several advantages. The smooth surface of glass capillary leads to excellent reproducibility within one sensor and between sensors of different batches. On the other hand, scalable sensor production can be easily achieved because of the small dimension. Tens of sensors can be simply constructed in one batch (Fig. S2). Additionally, the capillary sensor is entirely compatible with the trace volume of the sample (50 $\mu \mathrm{L}$ ). Hence, glass capillary is a worthy alternative for prevalent substrates such as paper, wafer and ITO glass slide (Kamon and Takeuchi 2018; Śmietana et al., 2020; Wang et al., 2019).

$\mathrm{Au}$ NSs have been developed as outstanding SERS nanosubstrates, where extraordinary field confinement and enhancement can be observed at the acute tips, which can thus act as supreme "hot spots" (Hrelescu et al., 2011; Indrasekara et al., 2014). In the current work, Au NSs were synthesized through one-pot, seedless, surfactantless, and green procedure. Accordingly, Au NSs can be easily obtained within 20 min at ambient temperature without needing shaking or stirring. From the TEM image (Fig. S3a), Au NSs are three-dimensional branched nanoparticles with several tips and have star shaped morphology (spherical core structures with protruding sharp tips). To prepare SERS sensors, the surface of glass capillary was first functionalized with amino groups (positively charged glass). Afterward, negatively charged Au NSs synthesized in HEPES buffer and electrostatically immobilized on the surface of the functionalized-capillary glass. This construction recipe can be easily accomplished in a general laboratory without complex equipment and thus, it is preponderant in commercial manufacture. Most importantly, it provides SERS substrates with a homogeneous SERS signal and large area. To assure the maximum density of Au NSs immobilized on the glass surface, the incubation time was optimized. As shown in Fig. S4, SERS intensity enhanced by passing time from 1 to $9 \mathrm{~h}$ and then fixed since all amino active sites of capillary glass saturated by
Au NSs. Thus, the capillary sensors gain the best SERS sensitivity at the optimum incubation time of $9 \mathrm{~h}$. SEM image (Fig. S3b) shows a high density of Au NSs evenly immobilized on the glass capillary with a low degree of aggregation; consequently, provide intense and homogeneous SERS signals which is the dominant merit of the electrostatically assisted surface-assembly approach. The negligible aggregation is probably attributed to the high concentration of Au NSs in solution before self-assembly and the polarization of Au NSs stimulated by the positively charged amino group of functionalized-capillary glass (Grabar et al., 1996; Wang et al., 2008).

Thereupon, the thin MIP shell was coated on Au NSs for preparation MIP-SERS sensor by using trypsin as a bioanalyte model. PDA can strongly stick on glass and gold surfaces through covalent and noncovalent bonds in physiological media and mild condition (Cheng et al., 2019; Lee et al., 2007a), while template protein molecules embedded in the 3D network of PDA without changing the spatial morphology. Fig. 2 displays the SEM images and Raman spectra of an optimal sensor at different preparation status. For the bare SERS sensor without PDA layer, DTTC molecules can freely attach to the Au NSs and the most intense SERS signal is observed (Fig. 2a and e). Fig. $2 b$ and e shows the sticking of the PDA layer containing trypsin template on the Au NSs surface ( $5 \mathrm{~h}$ polymerization time). In this condition, the accessibility of DTTC molecules to the Au NSs are limited and the signal vanished. After depleting of trypsin locus, the signal intensity recovered about $49 \%$ of bare sensor upon DTTC incubation, implying DTTC molecules permeated through imprinted cavities for the generation of sensing signal (Fig. 2c and e). The binding and removal of trypsin molecules from the PDA skeleton have no apparent effect on the SEM morphology of the PDA shell (Fig. 2b and c) proving the robustness of the PDA layer (Luo et al., 2013). For the construction of NIP sensor, no protein template was used, and Au NSs uniformly covered by dense PDA layer (Fig. 2d). Therefore, no specific binding site for protein and no pathway for DTTC flux exists. Accordingly, the NIP sensor was lack of signal (Fig. 2e) and considered as control.

The bare capillary glass, immobilized Au NSs on glass (capillary glass@Au NSs), and MIP-SERS sensor were characterized by crosssectional SEM, energy dispersive X-Ray (EDX), and contact angle. The capillary glass substrate has a very smooth surface (Fig. S5a). After immobilization of Au NSs, a layer of Au NSs contain star-shaped particles with high density was covered the capillary surface (Fig. S5b). From Fig. S5c, imprinted PDA layer buried Au NSs, and uniformly coated capillary@Au NSs substrate. Elemental composition of the samples was studied by EDX analysis. Emerging of Au peaks in the EDX spectra of capillary glass@Au NSs revealed that Au NSs successfully immobilized on the glass surface (Figs. S5d and e). Compared with capillary glass@Au NSs, MIP-SERS sensor has a higher content of C and O elements, which is attributed to the coated PDA layer (Fig. S5f). Atomic force microscopy (AFM) further utilized to investigate the materials' surface. Fig. S6a indicates, immobilization of Au NSs on the glass capillary provided an uneven surface. After the imprinting process, because of the uniform coating of the PDA layer and burial of Au NSs, the surface of MIP-SERS sensor became smoother (Fig. S6b). The hydrophilicity of the bare glass capillary, amino-functionalized glass, capillary glass@Au NSs, and MIP-SERS sensor was assessed by surface water contact angle (CA) measurement. As shown in Fig. S7, the CA degrees for the bare glass capillary, amino-functionalized glass, capillary glass@Au NSs, and MIP-SERS sensor are $53^{\circ}, 58^{\circ}, 34^{\circ}$, and $62^{\circ}$, respectively. All substrates are hydrophilic and the variation in CA degrees verified the successful modification of bare glass in each step.

The thickness of the PDA shell plays a crucial role in sensing. On one hand, the shell should provide enough distance between SERS substrate and adsorbed DTTC molecules on the outer shell of PDA layer, to prevent the generation of background SERS signal via shell-isolated nanoparticle-enhanced Raman spectroscopy (SHINERS) effect. On the other hand, it should not be too thick to preclude the penetration of DTTC through the vacant imprinted cavities to light up the SERS signal for 

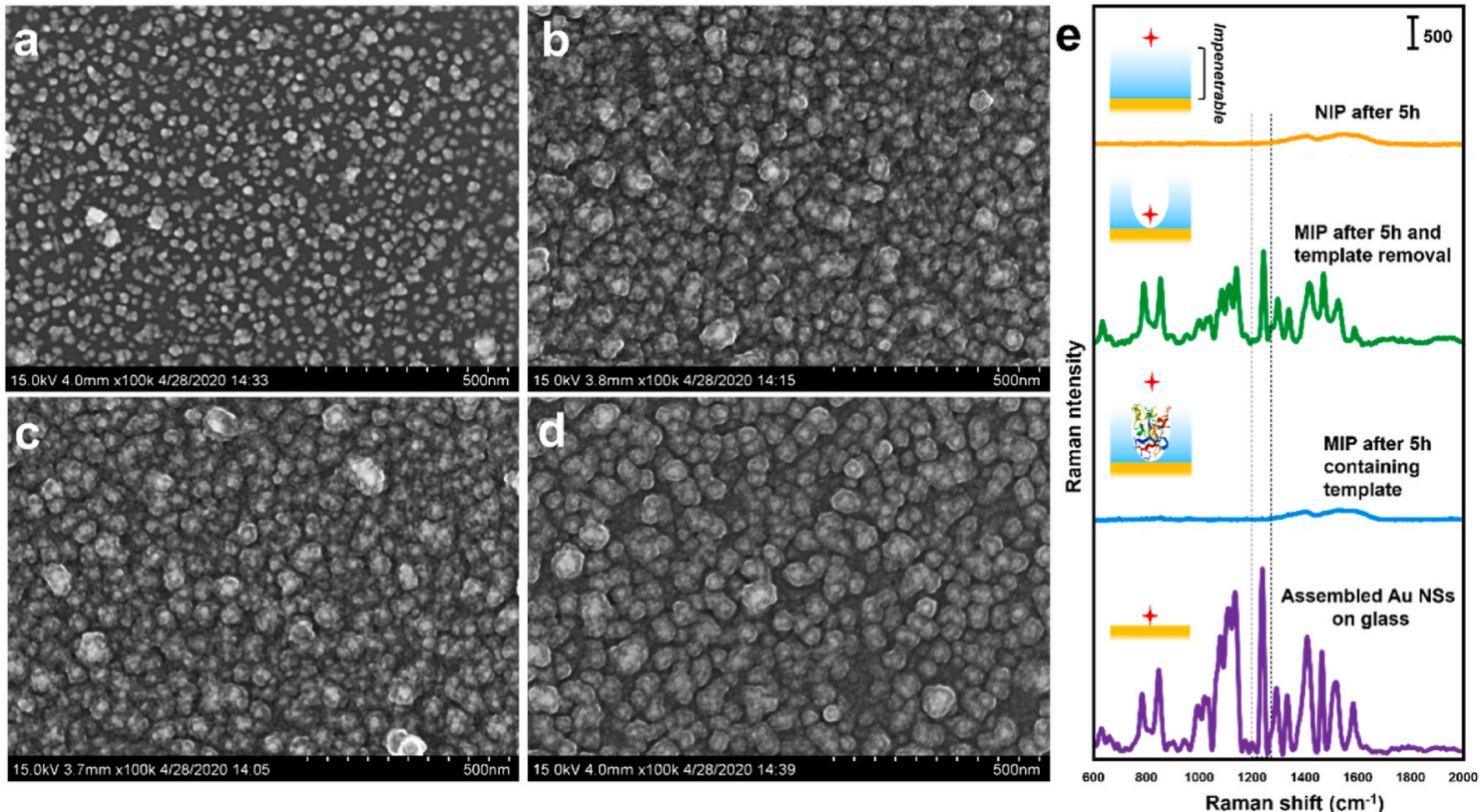

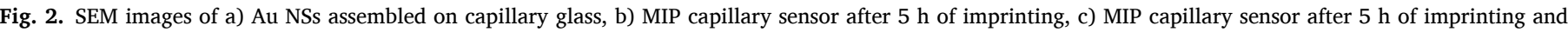

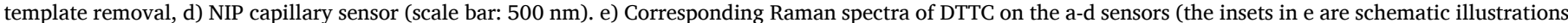

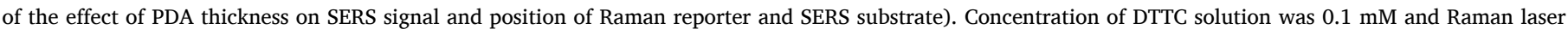
power was $100 \mathrm{~mW}$ in all experiments.

analyte sensing. For the optimization of PDA thickness, the background SERS intensities of sensors ( $1246 \mathrm{~cm}^{-1}$ Raman band of DTTC) preparing with varied polymerization times, were measured. The diagram of surface imprinting (polymerization) time versus SERS intensity and SEM images of corresponding sensors are depicted in Fig. S8. As seen, the signals decrease gradually with prolonged polymerization time. In the early hours ( $1 \mathrm{~h}$ and $3 \mathrm{~h}$ ), the PDA layers are provided SHINERS sensing effect (Li et al., 2010) of DTTC when the layer is very thin (less than 10 $\mathrm{nm}$ ) (Ye et al., 2017). After $5 \mathrm{~h}$, the signal totally disappeared, indicates PDA layer is thick enough to completely isolate the generation of DTTC signal and eliminate detection background. Hence, the optimal polymerization time is $5 \mathrm{~h}$.

\subsection{Analytical performance}

The optimization of sensor construction and detection protocol were
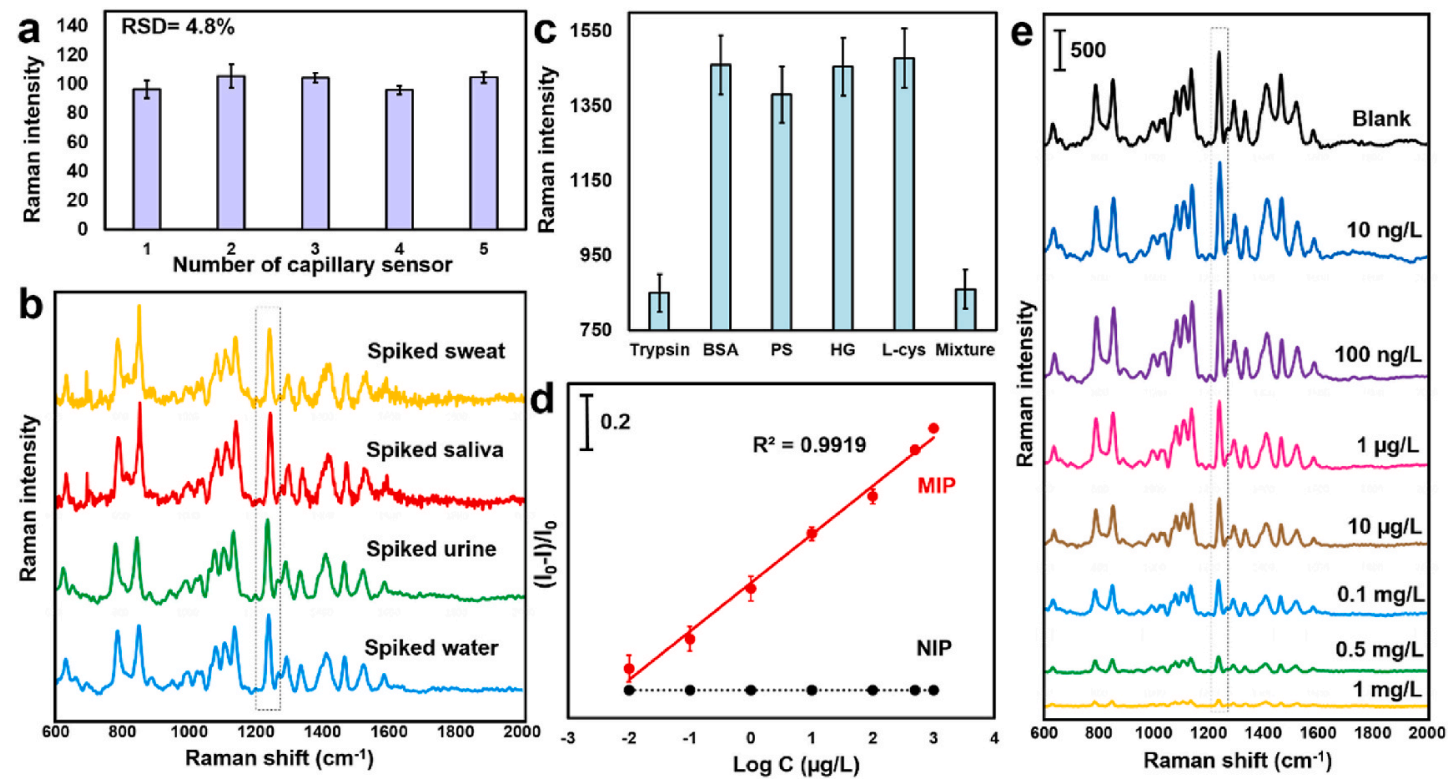

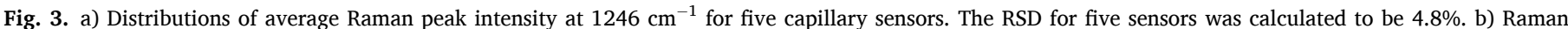

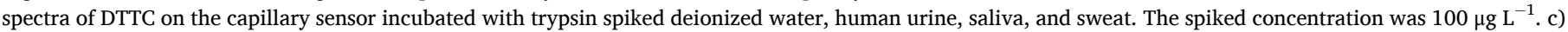

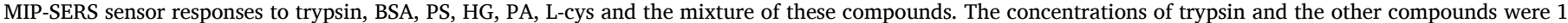

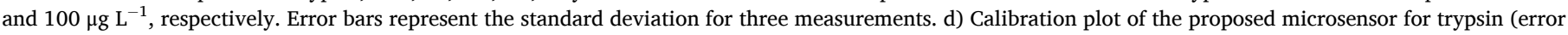


was $100 \mathrm{~mW}$ (except of a, $10 \mathrm{~mW}$ ) in all experiments. 
performed (details are present in Supporting Information). The analysis of proteins can simply be carried out at three stepwise: 1) specific protein recognition implemented by dipping the tip of the capillary sensor into the as-received biological sample and incubated, followed by dipping in deionized water to remove non-specific adsorption; 2) staining bonded protein by plunging detection zone of the sensor in CBBG solution; 3) smeared tip of the capillary sensor with DTTC solution and then SERS detection. All operational and sensing procedures were performed in less than $15 \mathrm{~min}$ and no pretreatment of the biological samples such as dilution, filtration and $\mathrm{pH}$ adjustment is needed (Fig. S9).

To test the possibility of quantitative analysis, the uniformity and reproducibility of the capillary sensor were firstly explored by measuring the SERS intensity of fifty random spots of five parallel sensors, and the results are indicated in Fig. 3a. Taking advantage of the uniformity of sensor surface, the relative standard deviation (RSD) of the fifty intensities of one sensor is less than $3.6 \%$, and the RSD of mean intensities from the five different sensors is $4.8 \%$, demonstrated supreme reproducibility amongst the capillary sensors for sensing.

The selectivity of the sensor was investigated in biological matrix, potential interferent proteins solutions, and metal ions. In this regard, the trypsin-sensing capability of the capillary sensor by comparing SERS intensities of DTTC for trypsin aqueous solution and spiked crude urine sample (without any pretreatment) at equal concentration was examined. As displayed in Fig. 3b, no sensible change in the two SERS intensities is observed implying matrix components don't interfere during trypsin measurement. For further specificity confirmation of the capillary sensor, the efficacy of small amino acid including, L-cysteine (Lcys), and large proteins such as BSA, PS, and HG as potential interferents were appraised. As illustrated in Fig. 3c, there is a negligible change in the SERS intensities for interferent species. Since, biological samples contain a high amount of metal ions, the effect of $\mathrm{Na}^{+}, \mathrm{K}^{+}, \mathrm{Ca}^{2+}$, and $\mathrm{Mg}^{2+}$ ions on the sensor performance was examined, and results are depicted in Fig. S10. The selected concentrations of the metal ions were the highest content that existed in urine, saliva, and sweat samples. The proposed MIP-SERS sensor maintains its high efficiency in complex metal solution with high concentration. Therefore, it can conclude the presence of other biological species with different sizes and functional groups and metal ions have no notable impact on trypsin sensing performance. The outstanding specificity can be a result of the synergistic participation of functionality and spatial shape in the recognition. Trypsin has numerous available functional groups, including carboxyl and amino which strongly interact with amino and hydroxyl sites of PDA. Besides, during polymerization, trypsin molecules are completely surrounded by the PDA network, so the created imprinted cavities are completely analogue to the shape and size of trypsin. Thus, only trypsin molecules can spatiality place into the imprinted cavities. Although intrusive species may be non-specifically adsorbed on the PDA layer, they can't fill imprinted cavities due to stereochemistry hindrance and meddle to specific recognition of trypsin.

In order to assess the trypsin capturing mechanism and the sensor performance, the influence of $\mathrm{pH}$ within the range of 5.0-9.0 was examined (a pH range that biological samples possessed). PDA contains amine groups and phenolic hydroxyl groups, which makes it potentially ampholytic or zwitterionic (Yu et al., 2010). Therefore, the surface charge of PDA changed by $\mathrm{pH}$ variation. The results demonstrated $\mathrm{pH}$ has a negligible effect on the MIP-SERS sensor performance (Fig. S11). Typically, functionality and spatial shape are simultaneously contributed to capturing target analyte by MIPs. According to the $\mathrm{pH}$ experiment, it can conclude that the dominant sorption mechanism of trypsin by PDA layer is the size and spatial shape of imprinted cavities, however, the influence of interactions between functional groups of PDA and trypsin should not be ignored.

Trypsin is a key to the non-invasive biomarker in urine for pancreatitis diagnosis. Hence, accurate detection of trypsin at the ultra-trace level in urine is very important for the early diagnosis of disease (SEE and SMITH 1991). The analytical characteristics of the capillary sensor were studied by building a matrix match calibration plot in the healthy human urine sample. As expected, the lowest concentration of trypsin had the highest SERS intensity and by increasing trypsin amount at $\mu \mathrm{g}$ $\mathrm{L}^{-1}$ levels, the signals suppressed obviously (Fig. 3d and e). Along with recognition event the DTTC flow path is partially blocked by trypsin and this proceeding is continued until complete blocking all DTTC flux and thereby can be utilized as a platform for trypsin quantification. Accordingly, the change in SERS intensity versus the logarithm of trypsin concentration has a linear response within the range of $0.01-1000 \mu \mathrm{g} \mathrm{L}^{-1}$ (Fig. 3d). In this case, the capillary sensor yielded an ultra-low limit of detection (LOD) of $4.1 \times 10^{-3} \mu \mathrm{g} \mathrm{L}{ }^{-1}$ which is one of the lowest values reported in the literature for trypsin (Table S1), qualifying for early diagnosis of pancreatitis. As a control experiment, the NIP capillary sensor lacked SERS signal for all tested concentrations (the black line in Fig. 3d), which further corroborates the source of SERS signals exclusively pertinent to the specific capturing of trypsin by imprinted cavities of the PDA layer.

The complexity of real sample matrix has a great effect on the reproducibility of the sensors. Since the developed MIP-SERS sensor is highly specific towards the target, we analyzed crude real samples without any pretreatment, avoiding several sample preparation steps mainly including centrifugation, dilution, $\mathrm{pH}$ adjustment, and etc., which have been performed for complex real sample analysis (Li et al., 2019b; Muhammad et al., 2017; Xing et al., 2019). In the following experiments, the urine samples were spiked with an exact amount of trypsin and the measurement was run. Excellent recoveries along with low RSD values are valid certificate to confirm high practical applicability of the sensor for clinical pancreatitis diagnosis (Table 1). To test the feasibility of protein biomarker analysis in other biological fluids, spiked saliva, and sweat samples were analyzed and results are presented in Fig. 3b and Table 1. It should be mentioned that the reproducibility of our MIP-SERS sensor is comparable with those of other developed MIP-SERS sensors (Castro-Grijalba et al., 2020; Lv et al., 2016). Relying on high accuracy and impressive anti-interference ability of the capillary MIP-SERS sensor, we can foresee that Raman indicator sensing mechanism may find plenty of room in wearable sensor to detect targets in sweat.

\subsection{Generality of the Raman indicator sensing mechanism and staining effect of proteins}

To affirm universal applicability of the proposed sensing strategy, more complex proteins with larger size and different subunits, including PS, BSA, and HG were selected as other models and corresponding sensors constructed. First, the role of CBBG staining in protein sensing was investigated. In this regard, after protein recognition, the sensors were immersed in the DTTC solution followed by SERS analysis (staining step not performed). For trypsin sensor, no significant difference observed in the results of linear range, and SERS intensity versus the logarithm of concentration has a linear response. In contrast with trypsin sensor, SERS intensities of all other protein sensors at different concentrations were very scattered and there was no reasonable

Table 1

Application of the proposed SERS capillary sensor to the determination of trypsin in spiked biological samples. $(n=3)$.

\begin{tabular}{lllll}
\hline Sample & Added $\left(\mu \mathrm{g} \mathrm{L}^{-1}\right)$ & Found $\left(\mu \mathrm{g} \mathrm{L}^{-1}\right) \pm \mathrm{SD}$ & Recovery $(\%)$ & RSD (\%) \\
\hline Urine 1 & 100 & $94 \pm 8.0$ & 94 & 8.5 \\
Urine 2 & 100 & $105 \pm 9.5$ & 105 & 9.5 \\
Urine 3 & 100 & $101 \pm 5.0$ & 101 & 4.9 \\
Saliva 1 & 100 & $103 \pm 8.1$ & 103 & 7.9 \\
Saliva 2 & 100 & $92 \pm 5.1$ & 92 & 5.5 \\
Saliva 3 & 100 & $98 \pm 7.2$ & 98 & 7.3 \\
Sweat 1 & 100 & $93 \pm 5.7$ & 93 & 6.1 \\
Sweat 2 & 100 & $104 \pm 6.5$ & 104 & 6.3 \\
Sweat 3 & 100 & $96 \pm 6.6$ & 96 & 6.8 \\
\hline
\end{tabular}


relationship between analytical signal and protein concentration (Fig. 4a). This phenomenon might be due to the inherent properties of selected proteins. PS, BSA, and HG have the larger size and much higher molecular weight $(\sim 34.5,66.5$, and $64.5 \mathrm{kDa}$, respectively) compared with trypsin $(\sim 24.0 \mathrm{kDa})$ (Fig. S12). Possibly, there is no null space (about the size of the DTTC) between interwoven peptide chains of trypsin. Consequently, without CBBG staining, DTTC flow can entirely block with locating trypsin in the imprinted cavities. For the other three proteins, empty pockets between peptide chains (more than the size of DTTC) can be a source of undesirable SHINERS signal generation. To overcome this problem, an ingenious CBBG staining procedure was applied. CBBG possesses two advantages. First, it is a general staining agent having high efficiency filling hydrophobic pockets, suitable for almost all kinds of protein. And second, it has not generated any interference SERS signal under $780 \mathrm{~nm}$ laser detection (Fig. S13). After CBBG staining, these spaces occupied and the penetration of DTTC blocked. In this condition, evaluating of SERS intensity versus protein concentration revealed that there is a linear relationship between the logarithm of concentration and intensity with supreme $\mathrm{R}^{2}$ (Fig. 4b) within the range of $1 \times 10^{-3}-1000 \mu \mathrm{g} \mathrm{L}^{-1}$. Ultra-low LODs of $0.6 \times 10^{-3}, 0.4 \times 10^{-3}$, and $0.4 \times 10^{-3} \mu \mathrm{g} \mathrm{L}^{-1}$ for PS, BSA, and HG sensors were achieved, respectively, which are comparable with those of other research works (Table S2-S4). NIP capillary sensors for all tested proteins lacked of SERS signal in all experiments and confirm the observed SERS intensity changes are ascribed to the specific binding of proteins to the MIP cavities in the PDA surface layer. Besides, the selectivity of each capillary sensor was confirmed by using other proteins and L-cys. The concentration of target analyte was 100-fold higher than that of other potential interferents. As depicted in Fig. 5, the proposed sensors exhibit an excellent specificity towards the target proteins over other interfering species even at higher concentrations. These promising results are valid evidence for the compatibility of Raman indicator sensing mechanism with a wide variety of biospecies targets with different molecular weight, size, and subunits and implying impressive universality of the capillary sensing platform.

\section{Conclusion}

In summary, a low-cost capillary SERS sensor with unrivalled efficiency, sensitivity, and specificity for accurate Raman-inactive protein biomarkers measurement is presented. The capillary sensor constructed by a coating mussel-inspired imprinted layer on the SERS substrate. The sensing response is the change in SERS intensity, arising from Raman reporter flux and approaching/attaching to the Au Ns through imprinted cavities of MIP as preset pathway, which blocked by specific recognition of the target protein. The empty pockets between the peptide chains of bonded protein were filled by CBBG Raman reporter as a site occupying reagent to prevent unfavorable DTTC adsorption. Owing to the benefit from the advantages of indicator sensing mechanism and excellent specificity of the capillary sensor, the proposed method offers ultra-low LOD down to $4.1 \times 10^{-3} \mu \mathrm{g} \mathrm{L}^{-1}$ for trypsin in the complex biological fluid samples without any pretreatment. The high generality of the proposed sensing mechanism is confirmed by using three different proteins as bioanalyte models. Because of economical, straightforward, and the repeatable construction manner of the capillary sensor accompanied by high throughput, facile and quick operational, as well as high potential for large-scale preparation, we expect such microsensor will be extensively applied in on-site clinical analysis. This strategy is universal and can be extended to the detection of a wide range of analytes such as macromolecules and pathogens.

\section{CRediT authorship contribution statement}

Maryam Arabi: Investigation, Validation, Writing - original draft. Abbas Ostovan: Validation, Data curation, Formal analysis, Writing original draft. Zhiyang Zhang: Investigation. Yunqing Wang: Methodology, Supervision, Funding acquisition, Writing - review \& editing. Rongchao Mei: Investigation. Longwen Fu: Resources. Xiaoyan Wang: Writing - review \& editing. Jiping Ma: Writing - review \& editing. Lingxin Chen: Supervision, Conceptualization, Funding acquisition.

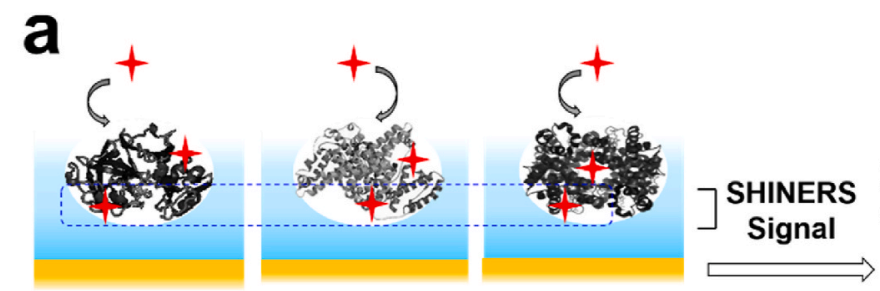

In the absence of CBBG
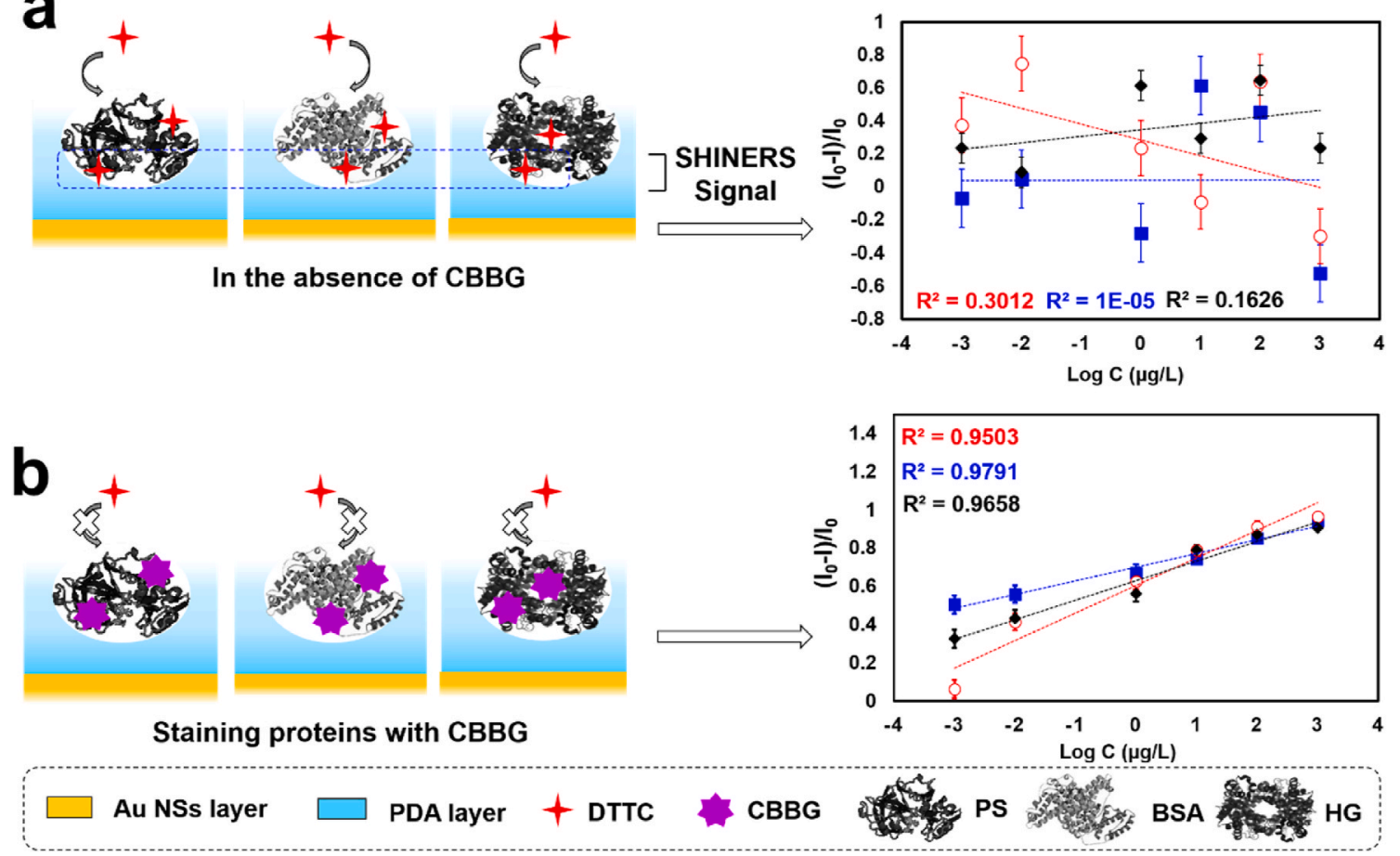

Staining proteins with CBBG

Fig. 4. SERS sensing mechanism for three different proteins with and without using CBBG. Corresponding calibration plots for PS $(\boldsymbol{\square})$, BSA $(\bigcirc)$, and HG ( $\bullet$ ) proteins. Error bars represent the standard deviation for five detections. Concentration of DTTC and CBBG solutions were $0.1 \mathrm{mM}$ and $0.6 \mathrm{mM}$, respectively. Raman laser power was $100 \mathrm{~mW}$ in all experiments. 

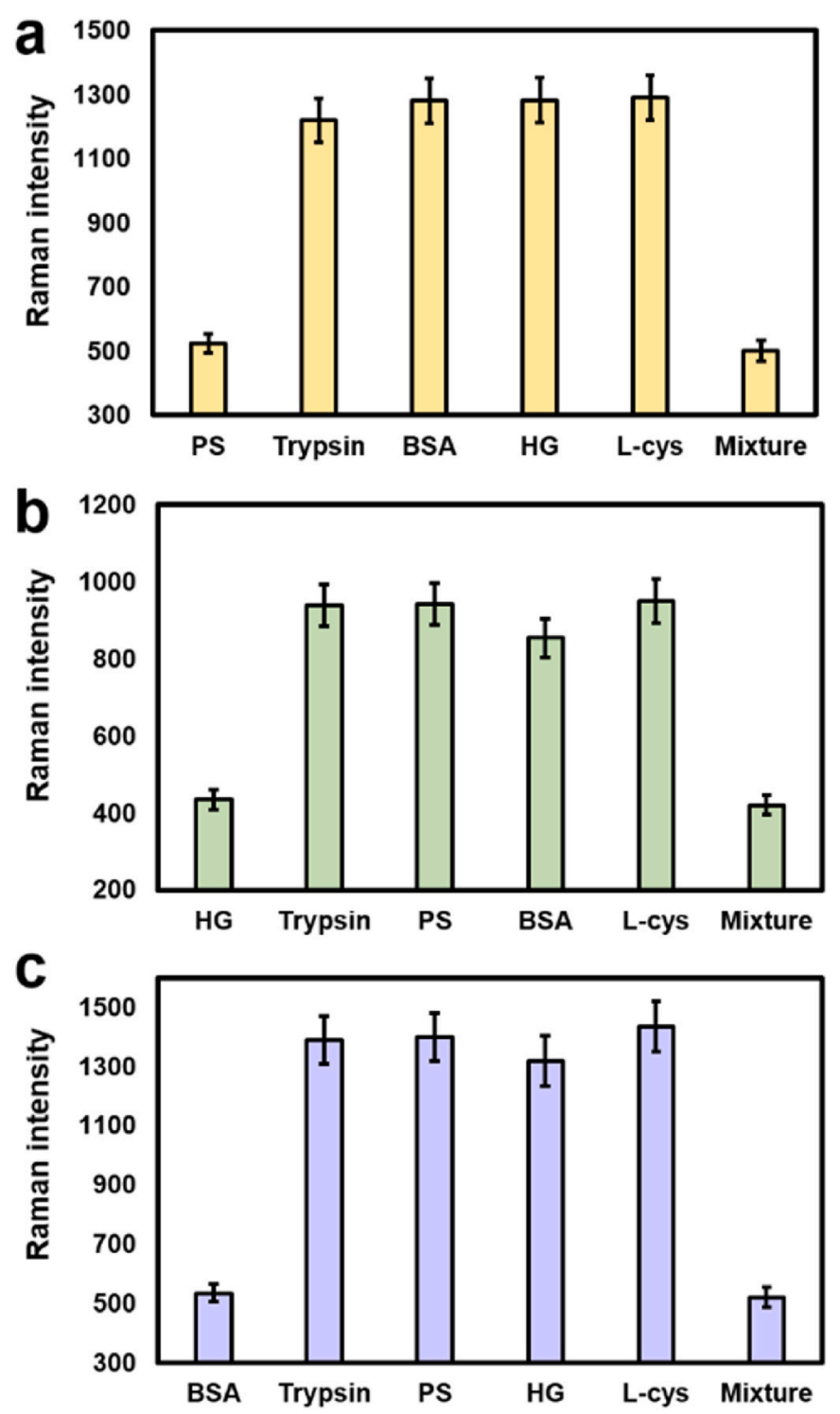

Fig. 5. Selectivity test for a) PS, b) HG, and c) BSA capillary sensors. The concentrations of target protein and the interferent compounds were 1 and 100 $\mu \mathrm{g} \mathrm{L}^{-1}$, respectively. Error bars represent the standard deviation for three measurements. Concentration of DTTC solution was $0.1 \mathrm{mM}$ and Raman laser power was $100 \mathrm{~mW}$ in all experiments.

\section{Declaration of competing interest}

The authors declare that they have no known competing financial interests or personal relationships that could have appeared to influence the work reported in this paper.

Acknowledgements.

This work was financially supported by the Chinese Academy of Sciences President's International Fellowship Initiative (2019PC0050, 2020PC0083), the National Natural Science Foundation of China (42076199, 21976209, 21804010, 22006162, 21976099 and 81573393), the Science and Technology Development Plan of Shandong Province of China (2019GSF108047), the Youth Innovation Promotion Association CAS (2017256), the Instrument Developing Project of the Chinese Academy of Sciences (YZ201662), the Technical Innovation Project of Instrument Function Development of the Chinese Academy of Sciences, and Taishan Scholar Project Special Funding (no. Ts20190962).

\section{Appendix A. Supplementary data}

Supplementary data to this article can be found online at https://doi. org/10.1016/j.bios.2020.112825.

\section{References}

Baker, M., 2015a. Antibody anarchy: a call to order. Nature 527, 545-551.

Baker, M., 2015b. Blame it on the antibodies. Nature 521, 274-276.

Castro-Grijalba, A., Montes-García, V., Cordero-Ferradás, M.J., Coronado, E., PérezJuste, J., Pastoriza-Santos, I., 2020. SERS-based molecularly imprinted plasmonic sensor for highly sensitive PAH detection. ACS Sens. 5, 693-702.

Chen, J., Lei, S., Xie, Y., Wang, M., Yang, J., Ge, X., 2015. Fabrication of highperformance magnetic lysozyme-imprinted microsphere and its NIR-responsive controlled release property. ACS Appl. Mater. Interfaces 7, 28606-28615.

Chen, L., Wang, X., Lu, W., Wu, X., Li, J., 2016. Molecular imprinting: perspectives and applications. Chem. Soc. Rev. 45, 2137-2211.

Chen, W., Fu, M., Zhu, X., Liu, Q., 2019. Protein recognition by polydopamine-based molecularly imprinted hollow spheres. Biosens. Bioelectron. 142, 111492.

Cheng, W., Zeng, X., Chen, H., Li, Z., Zeng, W., Mei, L., Zhao, Y., 2019. Versatile polydopamine platforms: synthesis and promising applications for surface modification and advanced nanomedicine. ACS Nano 13, 8537-8565.

Culver, H.R., Peppas, N.A., 2017. Protein-imprinted polymers: the shape of things to come? Chem. Mater. 29, 5753-5761.

Grabar, K.C., Smith, P.C., Musick, M.D., Davis, J.A., Walter, D.G., Jackson, M.A., Guthrie, A.P., Natan, M.J., 1996. Kinetic control of interparticle spacing in Au colloid-based surfaces: rational nanometer-scale architecture. J. Am. Chem. Soc. $118,1148-1153$.

Guo, X., Li, J., Arabi, M., Wang, X., Wang, Y., Chen, L., 2020. Molecular-imprintingbased surface-enhanced Raman scattering sensors. ACS Sens. 5, 601-619.

Han, X.X., Chen, L., Guo, J., Zhao, B., Ozaki, Y., 2010a. Coomassie brilliant dyes as surface-enhanced Raman scattering probes for protein - ligand recognitions. Anal. Chem. 82, 4102-4106.

Han, X.X., Xie, Y., Zhao, B., Ozaki, Y., 2010b. Highly sensitive protein concentration assay over a wide range via surface-enhanced Raman scattering of coomassie brilliant blue. Anal. Chem. 82, 4325-4328.

Hrelescu, C., Sau, T.K., Rogach, A.L., Jäckel, F., Laurent, G., Douillard, L., Charra, F., 2011. Selective excitation of individual plasmonic hotspots at the tips of single gold nanostars. Nano Lett. 11, 402-407.

Indrasekara, A.D.S., Meyers, S., Shubeita, S., Feldman, L.C., Gustafsson, T., Fabris, L., 2014. Gold nanostar substrates for SERS-based chemical sensing in the femtomolar regime. Nanoscale 6, 8891-8899.

Kamon, Y., Takeuchi, T., 2018. Molecularly imprinted nanocavities capable of ligandbinding domain and size/shape recognition for selective discrimination of vascular endothelial growth factor isoforms. ACS Sens. 3, 580-586.

Kao, Y.-C., Han, X., Lee, Y.H., Lee, H.K., Phan-Quang, G.C., Lay, C.L., Sim, H.Y.F. Phua, V.J.X., Ng, L.S., Ku, C.W., 2020. Multiplex surface-enhanced Raman scattering identification and quantification of urine metabolites in patient samples within 30 min. ACS Nano 14, 2542-2552.

Kumar, S., Bukkitgar, S.D., Singh, S., Singh, V., Reddy, K.R., Shetti, N.P., Venkata Reddy, C., Sadhu, V., Naveen, S., 2019. Electrochemical sensors and biosensors based on graphene functionalized with metal oxide nanostructures for healthcare applications. Chemistry 4, 5322-5337.

Lee, H., Dellatore, S.M., Miller, W.M., Messersmith, P.B., 2007a. Mussel-inspired surface chemistry for multifunctional coatings. Science 318, 426-430.

Lee, H., Lee, B.P., Messersmith, P.B., 2007b. A reversible wet/dry adhesive inspired by mussels and geckos. Nature 448, 338-341.

Li, C.-c., Wang, Z.-y., Wang, L.-j., Zhang, C.-y., 2019a. Biosensors for epigenetic biomarkers detection: a review. Biosens. Bioelectron. 144, 111695.

Li, J.F., Huang, Y.F., Ding, Y., Yang, Z.L., Li, S.B., Zhou, X.S., Fan, F.R., Zhang, W., Zhou, Z.Y., Ren, B., 2010. Shell-isolated nanoparticle-enhanced Raman spectroscopy. Nature 464, 392-395.

Li, W., Zhang, Q., Wang, Y., Ma, Y., Guo, Z., Liu, Z., 2019b. Controllably prepared aptamer-molecularly imprinted polymer hybrid for high-specificity and high-affinity recognition of target proteins. Anal. Chem. 91, 4831-4837.

Lim, W.Y., Goh, C.-H., Thevarajah, T.M., Goh, B.T., Khor, S.M., 2020. Using SERS-based microfluidic paper-based device ( $\mu \mathrm{PAD}$ ) for calibration-free quantitative measurement of AMI cardiac biomarkers. Biosens. Bioelectron. 147, 111792.

Luo, J., Jiang, S., Liu, X., 2013. Efficient one-pot synthesis of mussel-inspired molecularly imprinted polymer coated graphene for protein-specific recognition and fast separation. J. Phys. Chem. C 117, 18448-18456.

Lv, Y., Qin, Y., Svec, F., Tan, T., 2016. Molecularly imprinted plasmonic nanosensor for selective SERS detection of protein biomarkers. Biosens. Bioelectron. 80, 433-441.

Moura, S.L., Martín, C.G., Martí, M., Pividori, M.I., 2020. Electrochemical immunosensing of nanovesicles as biomarkers for breast cancer. Biosens. Bioelectron. 150, 111882.

Muhammad, P., Tu, X., Liu, J., Wang, Y., Liu, Z., 2017. Molecularly imprinted plasmonic substrates for specific and ultrasensitive immunoassay of trace glycoproteins in biological samples. ACS Appl. Mater. Interfaces 9, 12082-12091.

Pan, J., Chen, W., Ma, Y., Pan, G., 2018. Molecularly imprinted polymers as receptor mimics for selective cell recognition. Chem. Soc. Rev. 47, 5574-5587.

See, W.A., Smith, J.L., 1991. Urinary levels of activated trypsin in whole-organ pancreas transplant patients with duodenocystostomies. Transplantation 52, 630-633. 
Shetti, N.P., Bukkitgar, S.D., Reddy, K.R., Reddy, C.V., Aminabhavi, T.M., 2019a. Nanostructured titanium oxide hybrids-based electrochemical biosensors for healthcare applications. Colloids Surf., B 178, 385-394.

Shetti, N.P., Bukkitgar, S.D., Reddy, K.R., Reddy, C.V., Aminabhavi, T.M., 2019b. ZnObased nanostructured electrodes for electrochemical sensors and biosensors in biomedical applications. Biosens. Bioelectron. 141, 111417.

Shetti, N.P., Mishra, A., Basu, S., Mascarenhas, R.J., Kakarla, R.R., Aminabhavi, T.M., 2020. Skin-patchable electrodes for biosensor applications: a review. ACS Biomater. Sci. Eng. 6, 1823-1835.

Śmietana, M., Koba, M., Sezemsky, P., Szot-Karpińska, K., Burnat, D., Stranak, V., Niedziółka-Jönsson, J., Bogdanowicz, R., 2020. Simultaneous optical and electrochemical label-free biosensing with ITO-coated lossy-mode resonance sensor. Biosens. Bioelectron. 154, 112050.

Usta, D.D., Salimi, K., Pinar, A., Coban, I.l., Tekinay, T., Tuncel, A., 2016. A boronate affinity-assisted SERS tag equipped with a sandwich system for detection of glycated hemoglobin in the hemolysate of human erythrocytes. ACS Appl. Mater. Interfaces 8, 11934-11944.

Wang, J., Dai, J., Xu, Y., Dai, X., Zhang, Y., Shi, W., Sellergren, B., Pan, G., 2019. Molecularly imprinted fluorescent test strip for direct, rapid, and visual dopamine detection in tiny amount of biofluid. Small 15, 1803913.

Wang, Y., Qian, W., Tan, Y., Ding, S., 2008. A label-free biosensor based on gold nanoshell monolayers for monitoring biomolecular interactions in diluted whole blood. Biosens. Bioelectron. 23, 1166-1170.
Xie, J., Lee, J.Y., Wang, D.I., 2007. Seedless, surfactantless, high-yield synthesis of branched gold nanocrystals in HEPES buffer solution. Chem. Mater. 19, 2823-2830.

Xing, R., Wen, Y., Dong, Y., Wang, Y., Zhang, Q., Liu, Z., 2019. Dual molecularly imprinted polymer-based plasmonic immunosandwich assay for the specific and sensitive detection of protein biomarkers. Anal. Chem. 91, 9993-10000.

Yang, K., Li, S., Liu, L., Chen, Y., Zhou, W., Pei, J., Liang, Z., Zhang, L., Zhang, Y., 2019. Epitope imprinting technology: progress, applications, and perspectives toward artificial antibodies. Adv. Mater. 31, 1902048.

Yang, Q., Li, J., Wang, X., Peng, H., Xiong, H., Chen, L., 2018. Strategies of molecular imprinting-based fluorescence sensors for chemical and biological analysis. Biosens. Bioelectron. 112, 54-71.

Ye, J., Chen, Y., Liu, Z., 2014. A boronate affinity sandwich assay: an appealing alternative to immunoassays for the determination of glycoproteins. Angew. Chem. Int. Ed. 53, 10386-10389.

Ye, W., Huang, H., Yang, W., Wang, X., Ren, C., Hu, Q., Li, Y., Ren, B., 2017. Ultrathin polydopamine film coated gold nanoparticles: a sensitive, uniform, and stable SHINERS substrate for detection of benzotriazole. Analyst 142, 3459-3467.

Yu, B., Liu, J., Liu, S., Zhou, F., 2010. Pdop layer exhibiting zwitterionicity: a simple electrochemical interface for governing ion permeability. Chem. Commun. 46, 5900-5902. 